markably small coupling is the separation of the instanton into two "constituents" dubbed merons. ${ }^{20}$ The relevance of this fluctuation in the present context is that it leads to a dramatic increase in the effective dipole moment of the instanton and a correspondingly dramatic change in the properties of the instanton gas regarded as a polar medium. This fact will play a role in the considerations of the next speaker.

\section{§IX. Conclusions}

This is not the moment to announce definitive statements about the structure of QCD. What should be obvious from this lightning review is that the stimulus to our imagination provided by the discovery of the instanton has led to the further discovery of unexpected richness of structure in our favorite theory of the strong interactions. These new structures may, if we are lucky, provide the key to understanding at least the outlines of the complexities of hadron structure directly from the QCD field theory Lagrangian. The Proceedings of the next Rochester Conference should reveal whether this hope is wellfounded.

\section{References}

1. A. M. Polyakov: Phys. Letters 59B (1975) 82.
2. A. Belavin, A. Polyakov, A. Schwartz and Yu. Tyupkin: Phys. Letters 59B (1975) 85.

3. G. 't Hooft: Phys. Rev. D14 (1976) 3432.

4. C. Callan, R. Dashen and D. Gross: Phys. Letters 63B (1976) 334.

5. R. Jackiw and C. Rebbi: Phys. Rev. Letters 37 (1976) 172.

6. G. 't Hooft: Phys. Rev. Letters 37 (1976) 8.

7. S. Coleman: Erice Summer School Lectures, 1977.

8. S. Adler: Phys. Rev. 177 (1969) 2426; T. S. Bell and R. Jackiw: Nuovo Cimento 60A (1969) 47.

9. E. Witten: Phys. Rev. Letters 38 (1977) 121.

10. G. 't Hooft: unpublished.

11. A. Schwartz: Phys. Letters 67B (1977) 172; R. Jackiw and C. Rebbi: Phys. Letters 67B (1977) 189.

12. M. Atiyah and R. Ward: Comm. Math. Phys. 55 (1977) 117.

13. E. Corrigan, D. Fairlie, P. Goddard and R. Yates: Comm. Math. Phys. 58 (1978) 223.

14. L. Brown, R. Carlitz, D. Creamer and C. Lee: Phys. Letters 71B (1977) 103.

15. N. Andrei and D. Gross: "The Effect of Instantons on the Short Distance Structure of Hadronic Currents," Princeton Preprint.

16. C. Callan, R. Dashen and D. Gross: "Toward a Theory of the Strong Interactions," Princeton preprint, Aug. 1977.

17. D. Caldi: Pbys. Rev. Letters 39 (1977) 121.

18. R. Carlitz: Phys. Rev. D17 (1978).

19. D. Forster: Phys. Letters B (1977).

20. C. Callan, R. Dashen and D. Gross: Phys. Letters 66B (1977) 375.

\author{
D. J. Gross
}

Princeton University

\section{§I. Introduction}

In this talk I shall review the present stage of a program, carried out over the last few years by Callan, Dashen and myself, ${ }^{1,2}$ to construct a theory of the strong interactions. The theory is QCD, given by the Lagrangian

$$
\mathscr{L}=\begin{gathered}
1 \\
4 g^{2}
\end{gathered} \operatorname{Tr} F_{\mu_{\nu}} F^{\mu_{\nu}}+\sum_{F} \bar{\varphi}_{F}\left(i \not D-m_{F}\right) \varphi_{F}^{\prime},
$$

which describes colored quarks of various flavors $(F)$ and masses $m_{F}$, interacting via $\mathrm{SU}_{3}$ color gauge interactions. This candidate for a theory of the strong interactions is uniquely selected by its asymptotic freedom and the resulting explanation of the observed almost free short distance behavior of hadrons. In addition it incorporates the established symmetries of hadrons $\left(\mathrm{SU}_{3}\right.$ when $m_{u} \simeq m_{d} \simeq m_{s}$ and chiral $\mathrm{SU}_{2} \times \mathrm{SU}_{2}$ when $m_{u} \simeq m_{d} \simeq 0$ ), and would appear to be consistent with the 
phenomenological models of hadrons (the quark model, the string model, the bag model etc.) which have lent so much insight into hadronic structure.

The aim of our program is to directly derive all the properties of hadrons-masses, couplings and scattering amplitudes-directly from the microscopic dynamics of QCD.

The most difficult problem that emerges in such an attempt is related to the fact that QCD contains no adjustable ("relevant") parameters. The Lagrangian contains as free parameters the gauge coupling, $g$, and the quark mass parameters, $m_{F}$. However the quark masses are not relevant parameters in QCD. Indeed heavy quarks can be totally neglected when studying the properties of light quark bound states and light quark masses can, to a very good approximation, be neglected (i.e., chiral $\mathrm{SU}_{2} \times \mathrm{SU}_{2}$ is a good Nambu-Goldstone symmetry). Thus the only relevant parameter is $g$ itself, which, however, can be replaced by the renormalization scale parameter $\bar{\mu}$ which introduces the scale of lengths into QCD. In such a theory with no dimensional parameters the coupling is a running coupling whose magnitude depends on the momenta under consideration (in QCD, due to asymptotic freedom, $g \rightarrow 0$ for large momenta or short distances). If the theory is at all sensible it will produce dynamical masses of particles and the value of the coupling at the scale size characterizing those states, $g\left(m_{\text {hadron }}\right)$, will be a calculable number. Indeed all dimensionless parameters will be calculable!

This circumstance means that any small expansion parameter in QCD must be determined self-consistently and cannot be determined in advance. In our program the small parameter, which allows us to use semi-classical (WKB) approximations, is the gauge coupling, $g$, itself. However the use of such a small parameter is an assumption that can only be justified after constructing a complete description of the theory which produces hadrons.

The method we use is to systematically explore the relevant degrees of freedom of QCD, starting with small scale fluctuations and working our way out to the infra-red region. Small scale fluctuations, of size $\rho$ or momenta $1 / \rho$, are easy to treat thanks to asymptotic freedom, which ensures that the coupling characterizing such fluctuations is small

$$
x(\rho) \equiv \frac{8 \pi^{2}}{g^{2}(\rho)} \simeq 11 \ln \left(\begin{array}{c}
1 \\
\rho \bar{\mu}
\end{array}\right) .
$$

Thus small scale fluctuations can be treated by semi-classical methods and consist of:

a) Gaussian or perturbative fluctuations, which can be treated by ordinary perturbation theory, and yield an asymptotic expansion of physical quantities in powers of $g^{2} / 8 \pi^{2}$.

b) Tunneling fluctuations, which can be calculated by WKB techniques and yield an expansion in the "density of instations."

The relevant parameter characterizing this expansion is the fraction of space-time occupied by instantons up to some scale size $\rho_{c}$ :

$$
f=\int \frac{\rho_{c} \mathrm{~d} \rho}{\rho} C x^{6}(\rho) \mathrm{e}^{-x(\rho)} .
$$

As one considers larger scale fluctuations $g^{2}$ increases, leading to larger perturbative as well as non-perturbative effects. We have found that the non-perturbative effects rapidly dominate once $g^{2} / 8 \pi^{2} \geq 1 / 20$. These effects have been calculated for a variety of processes and we have shown, within the dilute gas approximation (DGA), that they

1) Yield an effective quark-antiquark potential that corresponds at large quark separation to a big coupling constant and mass renormalization. ${ }^{1,3}$

2) Yield large powerlike corrections to the short distance behavior of hadronic currents. ${ }^{4}$

3) Generate a mechanism for the dynamical breaking of chiral $\mathrm{SU}(2)$ and generate a dynamical quark mass.

However, these results are extremely sensitive to $\rho_{c}$ and cannot be quantitatively trusted. Indeed one must include fluctuations of all sizes and within the DGA there is no mechanism for suppresion of large instantons. In other words a naive application of the DGA implies that $f \gg 1$ (as $\rho_{c} \rightarrow \infty$ ) and therefore the DGA is incorrect-i.e., the vacuum is not well described as a dilute gas of instantons.

The new results reported below are the product of our recent study of the structure of the $Q C D$ vacuum taking into account instanton interactions. Briefly we find that the QCD vacuum can exist in two phases which can coexist in equilibrium in the presence of color fields provided by quarks. This then leads to 
a two-phase picture of hadrons similar, at a crude level, to the MIT bag model. In this picture the quarks live in a bubble immensed in the dense vacuum phase.

The phase which exists inside the bubble is a very simple phase, in which the coupling is small $\left(g^{2} / 8 \pi^{2} \sim 1 / 20\right)$ and the density of instantons is low $(f \sim .01$ in the bubble and $f \sim .1$ on its surface). This then explains why hadrons can still be regarded a simple collection of quarks with weak couplings even on the hadronic scale size. Furthermore we now have determined self-consistently the infra-red cutoff (i.e., the hadronic scale size) and can perform calculations with no cutoffs present. Thus, for example, non-perturbative effects due to instantons inside the bubble can be calculated using the formulae previously derived in the DGA, however the density of instantons in these formulae is not the spatially uniform perfect gas density, but rather the dynamiaclly perfect gas density, but rather they dynamically determined density of instantons in the hadronic bubble. This density contains a natural cutoff for large $o$, and is very dilute. Finally, we can also see how the formation of such a bubble shields the quarks from the large scale fluctuations of the vacuum outside the hadron. Thus the structure of hadrons might be quite insensitive to the detailed structure of the dense vacuum phase, whose effects to a large extent may be replaced by boundary conditions on the quark and gluon fields at the bubble boundary.

\section{§II. The Analog Gas of Permanent Magnetic Dipoles}

The vacuum to vacuum amplitude $Z=$ $\left\langle\mathrm{vac}\left|\mathrm{e}^{-i H T}\right| \mathrm{vac}\right\rangle$, which one studies in order to learn about the structure of the vacuum (hadrons, of course, are just low lying excitations of the vacuum), can usefully be regarded, after analytic continuation to Euclidean space, as the partition function for four dimensional non-abelian magnetostatics

$$
Z=\int\left(\mathscr{D} A_{\mu}\right) \exp \left\{\begin{array}{l}
-1 \\
4 g^{2}
\end{array} \int F_{\mu_{\mu}}^{\alpha} F_{\alpha}^{\mu^{\prime} \nu} \mathrm{d}^{4} x\right\} .
$$

Here $F_{\mu^{\mu}}$ is the (dual) magnetic field in four dimensional space and $g^{2}$ corresponds to $k T$. The properties of heavy quarks can be studied by evaluating the expectation value of the
Wilson loop operator $P \exp \left[i \oint_{L} A^{\prime \prime} \mathrm{d} x_{t}\right]$, which is equivalent to calculating the free energy of a fixed current loop.

The semi-classical evaluation of the functional integral, valid for small $g^{2}$, yields

$$
\begin{aligned}
Z= & \sum_{n_{+}, n_{-}} n_{+} ! n_{-} ! \\
& \times \exp \left[-S_{\mathrm{INT}}\left(\Omega_{i}^{ \pm}\right)\right],
\end{aligned}
$$

where the sum runs over all instanton-antiinstanton configurations, $\Omega_{i}$ labels their collective coordinates (position, scale size $\rho$ and group orientation) $n_{0}(\rho)$ is the activity

$$
n_{0}(\rho)={ }_{\rho^{4}}^{C} x^{6}(\rho) \mathrm{e}^{-x(\rho)},
$$

and $S_{\mathrm{IXT}}\left(\Omega_{i}^{ \pm}\right)$represents the interaction of the instantons. This can be regarded as the partition function, in the grand canonical ensemble of an interacting gas of solitons =instantons. If $S_{\text {INT }}$ is neglected one recovers the DGA.

We have studied the instanton-antiinstanton interaction and find that, to a large extent, they can simply be regarded as permanent magnetic dipoles. ${ }^{2}$ In fact as long as the density of instantons is less than .1-1.0 one can linearize the system and treat the dipoles as Abelian. The non-Abelian nature of the theory is only crucial in being responsible for the existence of finite action, topologically quantized permanent magnetic dipoles.

Thus an instanton field, $A_{t \prime}^{\alpha}=R_{\alpha a} \eta_{a t^{\prime \prime} \alpha} x^{\nu} \rho^{2} /$ $x^{2}\left(x^{2}+\rho^{2}\right)$ corresponds to a four dimensional magnetic dipole with a dipole moment $D_{\mu \nu}^{\alpha}=R_{\alpha a} \eta_{a \mu \nu} \rho^{2}$. Four dimensional dipoles are characterized by antisymmetric tensors, and come in two varieties, self-dual and antiself-dual, corresponding to instantons and antiinstantons. Their interaction with an external field $F_{\mu \nu}^{\mathrm{ext}}$ is given by $\left(2 \pi^{2} / g^{2}\right) D_{t_{\nu}}^{\alpha} F_{\mu^{\mu \nu}}^{\alpha(\operatorname{ext})}$, and their mutual interaction is given by $S_{\mathrm{INT}}=$ $\left(8 \pi^{2} / R^{4}\right) T_{i \alpha \alpha}(R) D_{\alpha \beta}^{1} T_{\beta \nu}(R) D_{\mu_{\nu}}^{2}$, where $T_{\mu \nu}(R)=$ $\delta_{\mu \nu}-2 \hat{R}_{t^{\prime}} \hat{R}_{\nu}$. The above interaction energy vanishes for two dipoles of the same duality (thus yielding a physical explanation of the non-interaction of instanton and instanton), and agrees with the instanton-antiinstanton interaction as calculated by other methods. ${ }^{1.5}$

The most important dynamical feature of such a gas of permanent dipole moments is that they generate a paramagnetic susceptibility, $\mu>1$. Thus if one defines $B_{\mu \nu}^{\alpha}=H_{\alpha \nu}^{\mu} \div$ 
$4 \pi^{2} M_{\mu \nu}^{\alpha}$, where $H_{\mu^{2 \nu}}$ is the field due to external sources (quarks) and $M_{k^{2}}^{\alpha}$ that due to the dipoles (instantons), then for weak fields $M_{\mu \nu}^{\alpha} \simeq \chi H_{\mu \nu}^{\alpha}$, and $B_{\mu \nu}^{\alpha}=\mu H_{\mu \nu}^{\alpha}$ where $\mu=1+4 \pi^{2} \chi$ $>1$. This corresponds to anti-screening of color and to "infrared slavery" or asymptotic freedom. The self-energy of a current loop (Wilson loop) in such a medium is increased, which corresponds to an increase in the coupling $g^{2}$ by a factor of $\mu$ (i.e.,

$$
\begin{aligned}
& \left.\left.\underset{4 g^{2}}{1} \operatorname{Tr} F F\right|_{\mathrm{vac}} \rightarrow \frac{1}{4 g^{2}} \operatorname{Tr} B \cdot H\right|_{\text {(medium) }} \\
& \left.\quad=\begin{array}{c}
1 \\
4 g^{2} \mu
\end{array} \operatorname{Tr}(B \cdot B)\right) .
\end{aligned}
$$

\section{§III. The Permeability of the Vacuum}

One of the key elements in our discussion of the QCD vacuum is the calculation of the permeability, $\mu$, of a vacuum which contains a given density $n(\rho)$ of instantons treated as dipoles with dipole moment $D_{\mu \nu}^{\alpha}=\rho^{2} R_{\alpha a} \eta_{a a^{\prime \prime \nu}}$ (which is valid for $f<1$ ). Here we adapt methods developed for three dimensional polar media (Onsager, 1937). We calculate the magnetization in the presence of an external field $F_{\mu \nu}^{\text {ext }}$, by summing the mean dipole moment of each instanton. The interaction of this instanton with the others is accounted for by treating them (outside some region) as a continuous medium of permeability $\mu$. This has the effect of making the field seen by the instanton equal to $2 \mu F_{\mu \nu}^{e x t} /(1+\mu)$ and affects the Boltzmann factor of the instantons. This then yields ${ }^{3}$

$$
\begin{aligned}
& \mu \equiv \sqrt{ } 1+\eta^{2}+\eta, \\
& \eta=\frac{\pi^{2}}{2} \int_{\rho}^{\mathrm{d} \rho} n(\rho) \frac{8 \pi^{2}}{g^{2}(\rho)} \rho^{4},
\end{aligned}
$$

an expression which should be valid up to densities of order 1 , and is exact to second order in the density.

This expression yields a very large permeability due to instantons. Thus if one use the perfect gas density, $n(\rho)=n_{0}(\rho)$ and includes all instantons up to $f=1$ then $\mu \approx 20$. Thus the QCD vacuum has, at the very least, a very large permeability. We actually believe that $\mu=-\infty$ in the ordinary vacuum state. This is what is required to have absolute confinement of color (as opposed to partial confinement where $\mu$ is large but finite and the quarks are very massive). A possible mechanism for this might be the ionization of merons. Merons are null-poles in our four dimensional gas, i.e., fields that fall off as $1 / r$ as $r \rightarrow \infty$. An instanton can be regarded as made of two merons separated by a distance $\Delta$ and possessing a dipole moment proportional to $\Delta^{2}$. The action required to produce such a pair is proportional to $S_{m}=-\ln \Delta\left(8 \pi^{2} / g^{2}(\Delta)+6.55\right)$ and thus the contribution of the merons to $\eta_{3}$ is proportional to ${ }^{2}$

$$
\underset{\substack{\text { meiron } \\ \text { pairs }}}{\eta_{\text {m }}} \sim \mathrm{d}^{4} \Delta \Delta^{4} \exp \left[-S_{m}(\Delta)\right],
$$

which begins to diverge for $g^{2}(\Delta) / 8 \pi^{2} \geq 1 / 17$. This suggests that the vacuum might be dominated by a phase consisting something like an ionized plasma of merons with $\mu=\infty$-i.e., a perfect paramagnet. This would then product aboslute confinement.

Finally we note that such a paramagnetic medium will exhibit a phenomenon analogous to magnetostriction-i.e., the density of the polar medium will be lowered in the vicinity of color fields. This effect is crucial in producing the hadronic bag, inside of which the strong color fields produced by the quarks suppress the density of large scale instantons.

\section{§IV. The First Order Phase Transition}

Due to the phenomenon of magnetostriction described above an extenal color field provides an infrared cutoff, suppressing large scale fluctuations. Thus for a large external field the structure of the vacuum will be very simple - corresponding to a dilute gas of instantons. As the magnitude of the extenal field is decreased the instanton density is increased and we find an instability in the system, due to both its paramagnetic susceptibility and the attractive dipole-dipole interactions. This then implies a first order phase transition and the existense of two phases of the QCD vacuum. ${ }^{2}$

To explore for this possibility we have calculated the free energy of the instanton gas in an external color field (which is to be thought of as being produced by heavy static quarks). The field is taken as constant, pointing in, say, the 3 direction $\left(E=\left|F_{03}\right|\right)$ and can be thought of as the electric field produced by static quarks widely separated 
along the $z$-axis. We then consider the free energy, $\tilde{F}(n, E)$, as a function of the instanton density, $n(\rho)$, and the electric field $E . \quad(\tilde{F}=$ $F-E \cdot D$, where $F$ is the free energy regarded as a functional of the field $D$ which is produced by external sources). The equilibrium states are those for which

$$
\left.\partial n(\rho)\right|_{\mathrm{fixed} E}=0 \rightarrow n(\rho)=n[E, \rho] .
$$

For thermodynamic statility we require $\delta^{2} F>0$, which requires $(\partial D / \partial E)>0$. If there exist for a given $E$ two solutions $n_{1}(\rho)$ and $n_{2}(\rho)$ then one can have two phases coexisting in equilibrium with $E$ tangential to the phase boundary. For this to be the case we must have equal pressures at the boundary, and this requires that ${ }^{2}$

$$
\tilde{F}\left(n_{1}(\rho), E_{c}\right)=\tilde{F}\left(n_{2}(\rho), E_{c}\right),
$$

which will determine the critical value of the field, $E_{c}$, at which the phases can coexist.

The free energy $\tilde{F}$ we take to be

$$
\tilde{F}=F_{0}(n(o))+F_{\mathrm{int}}(n(\rho))-\frac{1}{2} E \cdot D,
$$

where $F_{0}$ is the perfect gas free energy:

$$
F_{0}=2 \int \frac{\mathrm{d} \rho}{\rho} n(\rho)\left[\ln \begin{array}{c}
n(\rho) \\
n_{0}(\rho)
\end{array}-1\right],
$$

and $F_{\text {int }}$ contains the effect of the instantonantiinstanton interactions. The latter is calculated by a mean field theory calculation, which evaluates the change in chemical potential of a single instanton $\left(\partial F_{\text {INT }} / \partial n(\rho)\right)$ by putting it in a sphere of radius $R$ and treating the instantons outside the sphere as a continuous medium of permeability $\mu$. The radius $R$ is to be thought of as a hard core radius for the instanton and is taken to be the value of the instanton-antiinstanton separation at which the dipole-dipole interaction begins to soften considerably. We find that $R \simeq 2.2 \rho$. This calculation is exact to second order in the density (which is quite sufficient to exhibit the instability) and should be reliable for $f \leqq 1$. Finally we assume that the magnetization in the external field does not saturate (an assumption that we verify a posteriori) and that $E=\mu D$ with $\mu$ given in terms of $n(\rho)$ by the expression given above.

This then leads to

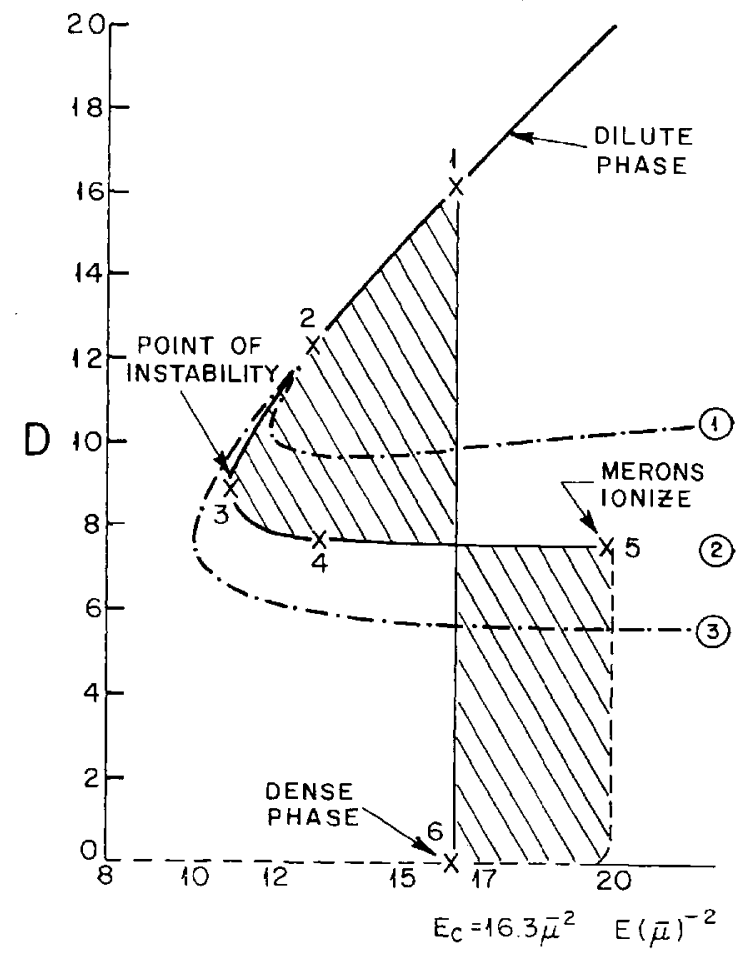

Fig. 1 .

$$
\begin{aligned}
n(\rho)= & n_{0}(\rho) \exp \left[-\frac{\pi^{2} E^{2}}{4\left(1+\mu^{2}\right)} x(\rho) \rho^{4}\right. \\
& \left.+6 x(\rho){ }_{\mu-1}^{\mu-1}\left(\frac{\rho}{R}\right)^{4}\right],
\end{aligned}
$$

which together with $\mu=\mu[n(\rho)]$ can be solved to evaluate $n(E, \rho)$. The result (the equation of state) is depicted in Fig. 1 which plots $D-(1 / \mu) E$ as a function of $E$ ( $D$ and $E$ are thermodynamic conjugate variables). The solid line (2) refers to the calculation done with $R=2.2 \rho$, whereas the dotted lines ( 1 and 3 ) refer to $R=.34 \rho$ and $.55 \rho$ respectively.

This calculation yields the following picture. For large $E$ the density of instantons is very low and $\mu \approx 1$ so that $D \approx E$. As $E$ is decreased the density and $\mu$ both increase leading to the curvature in $D(E)$. However we find an instability that shows up at point 3 at which point $\partial E / \partial D=0$. From point 3 to point 5 the system is unstable and a first order phase transition will actually occur at an earlier point (point 1). To determine the value of $E\left(E_{o}\right)$ at which the phase transition occurs one would have to see the $D(E)$ curve turn around again. However our approximations break down at point 5 , where the density becomes very large $\left(f_{5} \approx 1\right)$ and we are approaching the point at which merons might dissociate $\left(x_{5} \sim 15\right)$. The dotted line represents our conjecture as to the behavior 
of $D(E)$ due to this dissociation which would cause $\mu$ to rise rapidly and probably diverge. One then determines the point (point 1) at which the two phases are in equilibrium (i.e., exert equal pressures on the phase boundary) by the standard Maxwell construction. With the above choice of $R$ (as well as a choice of coupling constant renormalization which render $\left.n_{0}(\rho)=\left(.1 / \rho^{4}\right) x^{6} \mathrm{e}^{-x}\right)$ the dilute phase (point $1)$ is characterized by a very low density of instantons. In fact at point $1 \mu=1.02, f=$ .0014 and the dominant instantons have a size corresponding to $x=22.6$. This is indeed a simple phase. It is in equilibrium, for $E=$ $E_{o}=16.3 \bar{\mu}^{2}$. with a dense phase which has a large $\mu$ (in fact infinite if our conjecture is correct). The difference in free energies of these two phases is then given by $(1 / 2) E_{c}^{2} \equiv B$.

One can also easily check the validity of the assumptions that we make at various points along the curve. We find that these are good up to point 5 and excellent up to point 4 . In fact at the point of instability itself (point 3 ) the density of instantons is still very low $(f \sim .02)$ and $\mu$ is only 1.17 $x \simeq 18.7$. In fact a virial expansion would have been sufficient to establish the existence of the instability and the ensuing first order phase transition. It is the existence of this phase transition, and the highly (or perhaps infinite) paramagnetic susceptibility of the dense vacuum phase which will produce partial (or perhaps absolute) confinement of colored quarks and will lead to a picture of hadrons as a collection of quarks inside a dilute phase bubble in equilibrium with the dense vacuum phase.

\section{§V. The Heavy Quark Bag}

Given the above picture of the QCD vacuum one can derive a 'bag model' for heavy quark

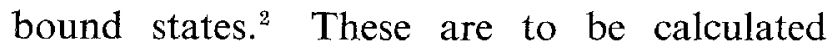
in a Born-Oppenheimer approximation, where one evaluates the interaction energy, $\varepsilon(R)$, between a quark-antiquark pair separated by distance $R$ and uses this as a static potential for determining the bound state energies. Higher order terms in powers of the inverse quark mass (spin-spin and spin-orbit interactions) can be systematically included. ${ }^{3}$

To evaluate $\varepsilon(R)$ one considers the vacuum expectation value of the Wilson loop operator, $\operatorname{Tr} P \exp i \oint_{L} A^{\mu} \mathrm{d} x_{\mu}$, for a loop $(L)$ of spatial extent $R$ and time extent $T$, as $T \rightarrow \infty$. However since the coupling is so small, inside the resulting bag, this is equivalent to treating the quarks as static, Abelian, sources of charge $Q g$ where $Q=\sqrt{ } 4 / 3$. In the normal dense vacuum state $\mu$ is infinite (or at least very large). Thus the color fields produced by the quarks cannot exist in this phase, and a bubble of dilute phase must be formed, in which the quarks exist, in equilibrium with the dense phase. Thus one must solve the linearized Yang-Mills equations for $E$ :

$$
\begin{aligned}
\bar{\nabla} \cdot \bar{D} & =g Q[\delta(\bar{x})-\delta(\bar{x}-\bar{R})], \bar{\nabla} \times \bar{E}=0, \\
\bar{D} & ={ }_{\mu}^{1} \bar{E},
\end{aligned}
$$

(where $\mu \approx 1$ is the permeability inside the bag) and $E_{\text {normal }}$ must vanish at the surface of the bag while $E_{\text {transverse }}=E_{c}$.

These equations are precisely those of the MIT bag model in the case of heavy quarks. Therefore we have provided a derivation of the MIT bag model (for static heavy quark bound states) as a zeroth order approximation in QCD.

In order to actually calculate a heavy quarkantiquark potential that would be used to predict the spectrum of charmonium or upsilonium one would have to improve this calculation. The effects of instantons inside the bag, the finite surface thickness (which we have estimated to be $\approx 10 \%$ of the radius of a typical hadron) and the resulting surface energy and tension, the effects of massless quarks and chiral symmetry breaking, would all have to be taken into account.

\section{§VI. The Light Quark Bag}

In order to treat light quarks one must know how they propagate in the instanton gas. We have argued $^{1}$ that instantons of rather low density induce dynamical chiral symmetry breaking and generate a dynamical quark mass. This will determine the boundary condition for the quark field at the surface of the bag. In fact inside the bag the density of instantons is so low that chiral symmetry breaking will not occur and the up and down quarks will be almost massless. However in the dense vacuum phase the quark acquire a very large dynamical mass (for small momentum). Thus the bag acts also as a mass bag, 
confining the quarks to lie inside simply because of their large mass outside the bag. If one approximates this situation by taking the quark mass outside the bag to the infinite one derives the MIT bag model boundary condition for the quark field.

Given our picture of the QCD vacuum we certainly expect that light quark bound states will be described as bubbles of dilute phase in equilibrium with the dense phase, where now the kinetic pressure of the quarks helps to balance the pressure on the surface of the bag. To give an adequate treatment of light quarks however one must go beyond our present methods which apply only to static configurations. If however we assume that the phase boundary responds slowly to the fluctuations of the quark and gluon fields we can develop a light quark bag along the same lines as the MIT bag. In fact the resulting equations are almost identical to those of the MIT bag model differing only in our treatment of chiral symmetry breaking. Since our vacuum (i.e., the dense phase outside the bag) is a Nambu-Goldstone vacuum it contains a massless excitation--the Nambu-Goldstone pion. This mode must be included in our treatment if we wish to preserve chiral symmetry. In fact it couples to the pseudoscalar quark density on the surface of the bag in precisely the right way as to lead to the conservation of the axial vector current. ${ }^{2}$ (It can be taken into account by introducing a phenomenological $\pi \equiv \bar{\varphi} \gamma_{s} \phi$ field). Thus in our picture the pion is not a bag state as the other low-lying hadrons but a Nambu-Goldstone excitation of the dense vacuum. If one considers a $0^{-}(I=1)$ q $\bar{q}$ bag-like state one finds that the attractive 4 fermion interaction induced by instantons (which is responsible for chiral symmetry breaking) tends to cause the bag to shrink to a size where it is no longer distinguishable from the dense vacuum phase.

\section{§VII. Conclusions and Outlook}

In summary we have seen that instantons and meron pairs are four dimensional (color) permanent magnetic dipoles, whose presence renders the QCD vacuum unstable against collapse to a new (strong coupling) phase which most likely exhibits perfect paramagnetism (absolute confinement). In the presence of an external field due to quarks this phase can exist in contact with a dilute phase where instanton effects are relatively small. This provides a (albeit nonrigorous) derivation in QCD of a picutre which, to first order, is similar to the MIT bag.

We can already at this stage see how the problem of confinement (which has to do with the properties of the dense vacuum phase) can be dissociated from the problem of hadronic structure (which has to do with the dynamics of the quarks in the dilute phase). Future progress will proceed in two directions. One must gain a greater understanding of the dense phase (learn how to deal with dense merons, include other relevant configurations, go beyond the semi-classical approximation if necessary, etc.) in order to learn whether $\mu$ is infinite or not. At the same time progress can already be made towards a calculation of the properties of hadrons. In fact for heavy quark bound states one might hope, within a relatively short period, to be able to directly calculate energy levels.

\section{References}

1. C. Callan, R. Dashen and D. Gross: Phys. Letters 63B (1976) 334; Phys. Letters 66B (1977) 375; Phys. Rev. D17 (1978) 2717.

2. C. Callan, R. Dashen and D. Gross: "A Theory of Hadronic Structure," I. A. S. Preprint, June 1978.

3. C. Callan, R. Dashen, D. Gross, F. Wilczek and A. Zee: Phys. Rev, to be published.

4. N. Andrei and D. Gross: Phys. Rev. D18 (1978) 468. 